\title{
Motivación en alumnos de Primaria en aulas con metodología basada en Proyectos
}

\author{
Alba Martín, Susana Rodríguez \\ Universidade da Coruña
}

\begin{abstract}
Resumen
El objetivo de la investigación era conocer las principales características motivacionales en relación a las metas académicas en una muestra de estudiantes de dos centros públicos, un centro con metodología tradicional y otro donde se trabaja por proyectos. En el ANOVA llevado a cabo se encontraron diferencias significativas entre los centros con respecto a la orientación a metas de evitación del trabajo y a la vertiente de aproximación de las metas de rendimiento. Se aportan conclusiones relevantes en torno a estas diferencias y se plantean líneas futuras de investigación.

Palabras clave: motivación académica, metas académicas, orientación a metas, aprendizaje basado en proyectos
\end{abstract}

\section{Introducción}

La motivación es una asignatura todavía pendiente en el sistema educativo español, según Valle, González \& Rodríguez (2006), la desmotivación académica es una de las cuestiones centrales explicativas de algunas situaciones problemáticas que se viven en los centros escolares; asimismo, Schleicher (2006), coordinador del Informe PISA de la OCDE, afirma que motivar al alumnado mediante contenidos útiles que le abran las puertas más allá de la escuela es lo que definirá el éxito del sistema educativo.

Si bien es cierto que la motivación dependerá de varios factores, uno de ellos sin duda es el desinterés hacia los contenidos que se enseñan en las clases mediante unas metodologías que han permanecido prácticamente iguales en los últimos años. Muchos docentes siguen centrando la dinámica de las clases en la transmisión y evaluación de los contenidos, contenidos prioritariamente teóricos y con pocas posibilidades de aplicación al mundo real que constituirán un "conocimiento inerte" (Valle et al., 2006). Este planteamiento instruccional se ha asociado en este estudio con una "educación más tradicional".

Es ampliamente reconocido que los métodos habitualmente asociados con la escuela tradicional, nucleados sobre el conocimiento abstracto, el libro de texto y la memorización de contenidos tienen difícil conectar con problemáticas e intereses reales del estudiante $y$ posiblemente tampoco conducen a profundizar en los contenidos de las materias, según Scheurich \& Higgins, citado en Dvorak (2012).

También Chávez (2003) señala que la práctica docente general se sustenta en la pedagogía tradicional, en el cual es el maestro el que decide qué y cómo enseñar, define las metas en el logro de los contenidos (no tanto en el aprendizaje de los alumnos) y los niños son considerados receptores del conocimiento que facilitan los libros de texto o el mismo docente: el alumno sigue relegado a actitudes pasivas. Esta misma autora considera que la intervención docente, es decir, cómo el docente concibe las metodologías didácticas, será la causa del conocimiento y del aprendizaje que propiciará en su alumnado. La metodología didáctica brinda la oportunidad al profesor de usar pautas para propiciar el aprendizaje y es la base del desarrollo de la práctica docente.

Como docentes, según Barron y Darling-Hammond (2008), estamos llamados a rediseñar el funcionamiento de las escuelas y a definir qué objetivos educativos deben prevalecer. Es necesario cambiar el planteamiento educativo, rediseñar sus prácticas instruccionales y hacer mayor énfasis en el desarrollo de las habilidades de aprendizaje por encima de los contenidos, crear conexiones entre el mundo real y contenidos de relevancia, así como fomentar el aprendizaje para toda la vida, el famoso "aprender a aprender" (Dvorak, 2012).

En esta línea, el modelo pedagógico de Proyectos de Trabajo o Aprendizaje Basado en Proyectos, (“ABP") (Project Based Learning, "PBL" en inglés), ha sido propuesto como como una herramienta que podría ser incorporada al sistema educativo para superar algunas de las limitaciones referidas antes (Blumenfeld et al., 1991), $\mathrm{y}$ ha sido objeto de discusión en diferentes reformas educativas, (Kilpatrick, 1918).

Esta metodología enraizada en los planteamientos de Dewey y Kilpatrick, que a su vez tendrían conexión con la filosofía de Maria Montessori (Thayer-Bacon, 2012), así como orígenes en común con los pioneros planteamientos de Bruner. Todos ellos contribuyeron en alguna medida a articular con sus experiencias esta metodología (Tobón, 2006), que podría ser definida como:

"una forma diferente de trabajar en la escuela, que privilegia la auténtica investigación estudiantil, a partir de interrogantes que los educandos consideren valiosos y que en buena parte hayan surgido de ellos mismos. Durante el desarrollo óptimo de un proyecto, los estudiantes exploran intereses, generan 
preguntas, organizan su trabajo, buscan información en diversas fuentes, indagan directamente en la realidad, ponen en movimiento sus concepciones y metaconcepciones, las confrontan con información nueva y las enriquecen o transforman, comunican resultados, hacen propuestas, eventualmente desarrollan acciones de cambio, etc.". (López y Lacueva, 2007, p.581)

El Aprendizaje Basado en Proyectos se inscribe en el marco constructivista, su rol fundamental es cultivar la atmósfera de cooperación y participación, el papel de profesor se caracteriza por ser guía y facilitador del proceso, desplazándose de un equipo a otro, observando, preguntando, respondiendo con sugerencias y ampliación de recursos, manteniendo y recordando las expectativas, orientando el camino de lo que conocer, discutir y modelar; en definitiva, el rol del profesor es estimular el proceso investigación de los alumnos (Vélez, 1998).

El alumno se convierte en un activo solucionador de problemas, se vuelve investigador y participante activo en toma de decisiones, por lo que es importante destacar la labor colaborativa y participativa con sus iguales (Pinzón, 2014). Según Lacueva (2008), éstos se ponen de acuerdo, con la ayuda del profesor, sobre una situación que persiguen investigar $\mathrm{y}$, a continuación: planifican, desarrollan, discuten y, por último, comunican su trabajo generalmente por equipos. De ahí que Vigotsky también sea fundamental en esta idea ya que desde sus planteamientos ha subrayado la importancia de las relaciones sociales en el desarrollo de las actividades mentales complejas, la internalización, transferencia y la zona de desarrollo próximo en el proceso de construcción del conocimiento, siendo el objetivo de aprendizaje el establecimiento de inferencias y las transferencias entre los conocimientos previos y las nuevas situaciones-problema que se plantean (Hernández, 2000).

Desde nuestro punto de vista, creemos necesaria más investigación acerca de cómo los estudiantes se comprometen con el trabajo a través en metodologías de este tipo (véase Blumenfeld et al. y Linnenbrick, citados en Dvorak, 2012). Asimismo, los estudiantes parecen obtener beneficios de la participación en ABP y, como recalcó Boaler (1998), son precisas nuevas investigaciones sobre los beneficios del Aprendizaje Basado en Proyectos en el proceso de aprendizaje de los estudiantes desde una perspectiva motivacional. En esta línea, un estudio de Wurdinger, Haar, Hugg, y Bezon en 2007 (citado en Dvorak, 2012) encontraron beneficios significativos en la motivación de los estudiantes derivados de la participación en Proyectos de Trabajo, estos mismos autores también reiteran la necesidad de más investigación en cómo los factores de la motivación interactúan en un ambiente de Aprendizaje Basado en Proyectos y su impacto en el logro académico de alumnos. También Thomas (2000), señaló como necesario estudiar los efectos sobre la motivación en el aprendizaje del estudiante y cómo los Proyectos de Trabajo pueden influenciar en sus orientaciones motivacionales.

Atendiendo a estas consideraciones, el presente estudio intenta profundizar en el conocimiento y la comprensión de cómo afecta el Aprendizaje Basado en Proyectos, (en comparación con una metodología más tradicional) sobre la motivación de los estudiantes. En este punto, cabe referir los tres componentes diferenciados por Pintrich y Degroot (1990): el componente de valor, el de expectativas y el componente afectivo. Este estudio se centrará en el componente de valor de la motivación: los motivos que tienen los estudiantes para realizar una tarea, ya que son estos motivos los que determinarán la importancia de la tarea de aprendizaje.

El componente de valor de la motivación integra, además del valor asignado a la tarea en sí mismo, las metas académicas entendidas como el propósito con el que el estudiante se compromete en la tarea. Las metas académicas definen el marco general por el cual el estudiante interpreta los contextos de logro, regula su motivación y adopta estrategias de aprendizaje (Dweck, 1986).

Inicialmente, desde la teoría de la orientación a metas, se diferenció entre metas de dominio, aprendizaje o centradas en la tarea de las metas ego-centradas, relativas a la capacidad o al rendimiento (Ames, 1992; Dweck, 1986; Nicholls, 1989). Diferentes estudios confirmaron la relevancia de las metas de logro en la medida que se asociaban de maneras cualitativamente diferentes al modo en el que los estudiantes evaluaban el éxito, procesaban la información o regulaban su comportamiento (véase, Kaplan \& Maehr, 2007).

Objetivos. El objeto del presente estudio era conocer en qué medida existen diferencias en la orientación a metas de los estudiantes que asisten a aulas con metodología tradicional y aquellos otros con educación por proyectos.

Hipótesis. Inicialmente presuponíamos la existencia de diferencias en orientación a metas entre los estudiantes que recibían educación tradicional y aquellos que asistían a clases con metodología por proyectos. En concreto, esperábamos que estos últimos estuviesen más orientados a metas de aprendizaje que los primeros.

\section{Método}

\section{Participantes}

La muestra sobre la cual se investigó se circunscribe a 110 estudiantes de $5^{\circ}$ de Educación Primaria de dos C.E.I.P diferentes, ambos de la provincia de A Coruña en Galicia. En uno de los centros se imparten las clases mediante Educación Por Proyectos y en el otro mediante una metodología fundamentalmente expositiva $\mathrm{y}$ centrada en el libro de texto.

Para la selección de la muestra, se tomó de referencia inicialmente un centro donde la metodología usada en el tercer ciclo de educación primaria fuese principalmente por proyectos. Ciñendo la búsqueda a la provincia de $\mathrm{A}$ Coruña y al último ciclo de Educación Primaria, de todos los centros consultados sólo se logró encontrar un centro en el cual se trabajaba por Proyectos. De este centro se recogió la muestra de 55 niños y niñas. En un segundo centro con una metodología principalmente tradicional 
que dio su permiso para llevar a cabo esta investigación, se recogió también una muestra de 55 niños y niñas.

Así, finalmente la muestra total resultó integrada por 110 estudiantes de $5^{\circ}$ de EP de dos escuelas públicas con una edad promedio de 10,28; de la cual el $46,4 \%$ son niñas y el $53,6 \%$ son varones

\section{Instrumento}

Para evaluar la orientación a metas, se ha utilizado la "Escala de Orientación a Metas" de Skaalvik (1997). Dicho instrumento establece diferencias entre tendencias de aproximación o de evitación tanto en las Metas de Aprendizaje como en las Metas de Rendimiento. La orientación a Metas de Aprendizaje se dirige a aprender, a resolver problemas y al desarrollo de capacidades. "Me gusta resolver los problemas trabajando duro" ejemplifica este tipo de orientación a metas. La vertiente de evitación de estas metas, denominadas por el autor de la escala como Metas de Evitación del Trabajo, recogerían los intentos de abordar las tareas académicas con el menor esfuerzo posible. Un ejemplo de esta dimensión sería: "en clase prefiero hacer lo menos posible". Las Metas de Rendimiento en su dimensión de Aproximación se dirigen a la demostración de capacidades y a la superación de los demás. Un ítem que ejemplificaría esta orientación sería: "es importante para mí saber hacer tareas que otros compañeros no saben". La vertiente de evitación de estas metas de rendimiento se caracteriza por evitar ser juzgado negativamente por los demás "lo peor de cometer errores en clase es que tus compañeros pueden darse cuenta".

Algunos términos del cuestionario han sido adaptados a un vocabulario más asequible para niños de tercer ciclo de Educación Primaria.

Para evaluar el grado en el que estos ítems medían constructos multidimensionales, sometimos los datos a un análisis factorial, usando como método de extracción el análisis de componentes principales y como método de rotación Normalización Varimax con Kaiser.

La Medida de adecuación muestral de Kaiser-Meyer-Olkin, es de .723, y la prueba de esfericidad de Bartlett, tiene un nivel de significación resultante de .000 . El análisis factorial resultante, permitió diferenciar los cuatro factores de la propuesta inicial para la Escala de Orientación a Metas por Skaalvik (1997). La varianza explicada conjuntamente por los cuatro factores extraídos alcanza el 53.74\% del total. Las respuestas a cada uno de los cuestionarios están categorizadas en una escala tipo Likert del 1 (nunca) al 5 (siempre).

\section{Procedimiento}

Es una investigación mediante encuesta $\mathrm{y}$, por tanto, descriptiva y no experimental, con el objetivo de caracterizar las relaciones entre las variables de esta investigación.

La recogida de información, se inició con la solicitud a los centros escolares de su participación anónima a través de varios emails a los directores de centros y llamadas telefónicas. En todo momento se informó del objetivo de esta investigación, de la estructura y contenido de los cuestionarios (de los cuales se les envió uno de ejemplar por correo electrónico), del tiempo requerido para su aplicación, en qué curso sería preferible y, finalmente, se dejó constancia de la disponibilidad a entregarles información con los resultados obtenidos una vez finalizado el estudio. Finalmente, se les entregó un consentimiento informado de confidencialidad en base a la Ley Orgánica 15/1999, de 13 de Diciembre, de Protección de Datos de Carácter Personal, donde se les aseguró el anonimato tanto de los centros como de los datos de los participantes.

La aplicación de la escala fue realizada en un único momento temporal en cada uno de los centros educativos, aplicada de forma colectiva para cada grupo en su aula correspondiente, utilizando unos 30 minutos aproximadamente para la realización del cuestionario. En ambos centros se realizó el estudio en la primera parte de su jornada escolar, entre las 10.00 y las 13.00 horas. A todos los participantes se les comunicó que los resultados de la investigación no iban a ser utilizados con fines diferentes a los ya descritos en la misma.

En el momento de la aplicación a todos los grupos se les insistió en la importancia de la sinceridad, en la no existencia de respuestas correctas o incorrectas y en la necesidad de contestar a todas y cada una de las preguntas.

Técnicas de análisis de datos. Los análisis se llevaron a cabo con el programa estadístico SPSS15.0 versión para Windows.

Se realizaron diversos análisis descriptivos, correlaciónales, ANOVA de un factor y un análisis factorial del cuestionario utilizado en esta investigación.

En primer lugar, como ya se ha explicado, se procedió al estudio de los ítems de la escala usando indicadores de tendencia central (media), indicadores de dispersión (desviación típica) e indicadores de distribución (asimetría y curtosis). Además del análisis factorial, se recurrió al ANOVA de un factor para conocer las diferencias entre los colegios en orientación a metas. Se utilizó como factor el centro y funcionando como variables dependientes, las cuatro metas académicas.

\section{Resultados}

Para identificar las posibles diferencias en la orientación a metas académicas entre los estudiantes del centro con una educación tradicional (a partir de ahora, Centro 1) y el centro con una metodología basada en Proyectos de Trabajo (Centro 2) se llevó a cabo un ANOVA de un factor (Tabla 1).

Tabla 1.

Diferencia en Metas Académicas en función del centro

\begin{tabular}{lccccccc}
\hline \multicolumn{7}{c}{ Centro1 } & \multicolumn{7}{c}{ Centro 2 } \\
\hline Metas & \multirow{2}{*}{$M$} & \multirow{2}{*}{$D T$} & $M$ & $D T$ & & & \\
Académicas & & & & & $F$ & sig & $\eta^{2}$ \\
\hline M_Evita_Rend & .0547 & .950 & -.0547 & 1.053 & .327 & .569 & .003 \\
M_Aprox.Rend & -.259 & 1.057 & .259 & .874 & 7.860 & .006 & .068 \\
M_Aprendizaje & -.158 & 1.022 & .158 & .961 & 2.93 & .098 & .025 \\
M_Evita_Trabajo & .269 & 1.099 & -.269 & .815 & 8.523 & .004 & .073
\end{tabular}

$\mathrm{M}=$ Media; $\mathrm{DT}=$ Desviación Típica; $\Pi^{2}=$ Eta cuadrado 
Escala de medida de las Metas Académicas: 1 = nunca, hasta $5=$ siempre.

A través del ANOVA, tal y como se observa en la tabla 1, se encontraron diferencias significativas en dos de las metas académicas: Evitación del trabajo y metas de aproximación al rendimiento. El centro con una metodología tradicional o expositiva (Centro 1) se caracteriza por un predominio de metas de evitación de trabajo.

El ANOVA realizado también nos permite constatar diferencias entre ambos grupos en cuanto a las Metas Aproximación al Rendimiento, siendo las puntuaciones medias obtenidas por el Centro 2 significativamente más altas.

En los otros tipos de metas no se observan diferencias significativas.

\section{Discusión}

Los resultados de este trabajo constatan la necesidad de abordar la orientación a metas diferenciando su vertiente de aproximación y evitación (Rodríguez et al., 2001). La vertiente de aproximación a las Metas de Aprendizaje se caracterizaría por una fuerte implicación, esfuerzo y dedicación, mientras que en su orientación de evitación podríamos remitirnos a una baja dedicación de esfuerzo y a la evitación del trabajo y compromiso académico (Pintrich, 2000; Skaalvik, 1997).

La literatura pone de manifiesto (véase, Blumenfeld et al., 1991; Chávez, 2003; Tobón, 2006) que ABP aumenta la motivación, el estudiante adquiere el gusto por aprender y por ahondar en el objeto de estudio y, en este sentido, los resultados de este trabajo corroboran que, frente a los que trabajan por proyectos, los estudiantes que asisten a aulas con metodología tradicional podrían estar más inclinados a optar por asignaturas en las que no haya que trabajar, evitando las materias más difíciles y con gran interés por evitar trabajar en casa y hacer lo menos posible en clase.

Por otra parte, diferenciando también las vertientes de aproximación/evitación en la orientación a metas relativas al rendimiento (Rodríguez et al., 2001), los resultados de este trabajo constatan que los estudiantes que asisten a aulas donde se trabaja por proyectos podrían estar más motivados por hacerlo mejor que los demás, conseguir notas más altas o saber hacer cosas que otros compañeros no saben. Dicho de otro modo, los estudiantes de $5^{\circ}$ de primaria del centro 1 -con metodología más tradicional- parecen menos preocupados por las notas o por demostrar sus habilidades que los estudiantes de esta edad que asisten al centro 2 donde se trabaja por proyectos.

Más allá de las posibilidades que nos permiten los datos del presente estudio, se podría inferir la incidencia motivacional de claves contextuales relativas a las metodologías adoptadas en ambos casos. Si es así, la metodología basada en proyectos puede promover un continuo funcionamiento y demostración de competencia al realizar la mayoría de las actividades en equipos y grupos de trabajo, donde cada uno hace las aportaciones al grupo que considera oportunas. Estos resultados nos llevan a sugerir la posibilidad de que los estudiantes que trabajan por proyectos acaben desarrollando una mayor metaconciencia en torno sus potenciales y limitaciones, y sería de interés conocer si, en este caso, entra en juego antes la comparación social como mecanismo evaluativo.

En este punto cabe destacar, como hacía Kaldi, Filippatou y Govaris (2011), la necesidad de que los estudiantes que trabajan por proyectos sean instruidos específicamente en trabajo cooperativo de modo que desarrollen la comprensión del propósito del grupo y la necesidad de apoyarse y ayudarse entre ellos (Sharan and Shaulov 1990). El aprendizaje cooperativo, enseñado explícitamente, no sólo favorece la resolución de los problemas, sino que también parece incrementar la motivación intrínseca de los alumnos hacia el trabajo académico (Gillies \& Ashman, 1996). Puede ser también de interés retomar la interpretación de estos resultados a la luz de la revisión desarrollada por Harackiewicz, Barron, Pintrich, Elliot \& Thrash (2002) donde se remarca la importancia de diferenciar las vertientes de aproximación y evitación de las metas de rendimiento identificando las formas en que estas metas pueden combinarse con la orientación al aprendizaje, lo que nos remite a la perspectiva de múltiples metas Valle, Cabanach, Núñez, González-Pienda, Rodríguez y Piñeiro, I., 2003). Valle, Núñez, Cabanach, Rodríguez, González-Pienda, y Rosário, 2009 y Valle, Rodríguez, Cabanach, Núñez, González-Pienda, y Rosário, 2010).

No podemos finalizar sin referirnos a las limitaciones del trabajo que presentamos aquí. Cabe indicar en primer lugar las limitaciones inherentes al tipo de diseño transversal que nos obligan a ser cautelosos con las relaciones especificadas entre las variables. Por otra parte, también somos conscientes de las limitaciones en el tamaño y selección de la muestra que nos exigen cautela en la generalización de los resultados de un trabajo llevado a cabo mediante encuesta por cuestionario.

\section{Referencias}

Ames, C. (1992). Classrooms: goals, structures, and student motivation. Journal of Educational Psychology, 84, 261-271.

Barron, B. \& Darling-Hammond, L. (2008). Teaching for meaningful learning. En Barron, B. \& Darling-Hammond, L., Pearson, P.D., Schoenfeld, A.H., Stage, E.K. \& Zimmerman, T.D. (Ed.), Powerful Learning: What We Know About Teaching for Understanding (pp. 11-70). San Francisco: John Wiley \& Sons Inc.

Blumenfeld, P.C., Soloway, E., Marx, R.W., Krajcik, J.S., Guzdial, M \& Palincsar, A. (1991). Motivating Project-Based Learning: Sustaining the Doing, Supporting the Learning. Educational Psychologyst, 23 (3 \& 4), 369-398.

Boaler, J. (1998). Open and Closed Mathematics: Student Experiences and Understandings. Journal for Research in Mathematics Education, 29, 41-62. http://dx.doi.org/10.2307/749717 
Chávez, A. (2003). El Método de Proyectos: una opción metodológica de enseñanza en primer grado de Educación Primaria. (Tesis inédita de maestría). Universidad Pedagógica Nacional. Culiacán Rosales, Sinaloa. http://www2.sepdf.gob.mx/proesa/archivos/p royectos/guia_general/metodo_proyectos_upn.pdf

Dvorak, R. (2012). Motivation towards learning: A study of Alberni District Secondary School's Project Based Learning Grade 9 Program. (Tesis Inédita de Maestría). Faculty of Education. Vancouver Island University. http://viuspace.viu.ca/bitstream/handle/10 613/2011/Dvorak.pdf?sequence $=1$

Dweck, C. S. (1986). Motivational processes affecting learning. American Psychologist, 41(10), 1040-1048.

Gillies, R.M., \& Ashman, F. (1996). Teaching collaborative skills to Primary School children in classroom-based work groups. Learning and Instruction, 6(3), 187-200.

Harackiewicz, J.M., Barron, K.E., Pintrich, P.R., Elliot, A.J. \& Thrash, T.M. (2002). Revision of achievement goal theory: Necessary and illuminating. Journal of Educational Psychology, 94(3), Sep 2002, 638-645. http://dx.doi.org/10.1037/0022-0663.94.3.63 8

Hernández, F. (2000). Los proyectos de trabajo: la necesidad de nuevas competencias para nuevas formas de racionalidad. Educar, 26, 39-51.

Kaldi, S., Filippatou, D y Govaris, C. (2011). Project-based learning in primary schools: effects on pupils' learning and attitudes. Education, 39(1), 35-47.

Kaplan \& Maehr, (2007). The Contributions and Prospects of Goal Orientation Theory. Educational Psychology Review, 19, 141-184.

Kilpatrick, W.H. (1918). The project method. Teacher's College Record, 19, 319-335.

Lacueva, A. (2008). Integrar para educar mejor: posibilidades y exigencias. Investigación en la escuela, 66, 43-53.

López, A.M. \& Lacueva, A. (2007). Enseñanza por proyectos:una investigación-acción en sexto grado. Revista de educación, 342, 579-604.

Nicholls, J. G. (1989). The competitive ethos and democratic education. Cambridge, MA: Harvard University Press.

Pintrich, P.R. (2000). An Achievement Goal Theory Perspective on Issues in Motivation Terminology, Theory, and Research. Contemporary Educational Psychology, 25, 92-

104. http://dx.doi.org/10.1006/ceps.1999.1017

Pintrich, P.R., \& De Groot, E.V. (1990). Motivational and Self-Regulated Learning Components of
Classroom Academic Performance. Journal of Educational Psychology, 82(1), 33-40.

Pinzón, R. J. (2014). English teaching through project based learning method, in rural area. Cuadernos de Lingüística Hipánica, 23, 151-170.

Rodríguez, S., Cabanach, R., Piñeiro, I., Valle, A., Núñez, J.C. \& González, J.A. (2001). Metas de Aproximación, Metas de Evitación y Múltiples Metas Académicas. Psicothema, 13(4), 546-550.

Schleicher, A. (2006), The Lisbon Council Policy Brief: The Economics of Knowledge. Lisbon Council, 1, 2-16.

Sharan, S., \& A. Shaulov. 1990. Cooperative learning, motivation to learn and academic achievement. In Cooperative learning theory and research, ed. S. Sharan, 173-202. New York: Praeger.

Skaalvik, E. M. (1997). Self-enchancing and self-defeating ego orientation: Relations with task and avoidance orientation, achievement, self-perceptions, and anxiety. Journal of Educational Psychology, 89, 71-81.

Thayer-Bacon, B. (2012). Maria Montessori, John Dewey, and William H. Kilpatrick. Education and Culture, 28 (1), 3-20.

Thomas, J.W. (2000). A Review of research on Project-Based Learning. San Rafael, C.A: The Autdesk Foundation.

Tobón, S. (2006). Método de trabajo por proyectos. Madrid: Uninet.

Valle, A., Cabanach, R. G., Núñez, J. C., González-Pienda, J.A., Rodríguez, S. y Piñeiro, I. (2003). Multiple goals, motivation and academic learning. British Journal of Educational Psychology, 73, 71-87.

Valle, A., González, R. \& Rodríguez, S. (2006). Reflexiones sobre la motivación y el aprendizaje a partir de la Ley Orgánica de educación (L.O.E.): “Del dicho al hecho...”. Papeles del psicólogo, 27(3), 135-138

Valle, A., Núñez, J. C., Cabanach, R. G., Rodríguez, S. González-Pienda, J.A. y Rosário, P. (2009) Perfiles motivacionales en estudiantes de secundaria: análisis diferencial en estrategias cognitivas, estrategias de autorregulación y rendimiento académico. Revista Mexicana de Psicología, 26, 113-124.

Valle, A., Rodríguez, S., Cabanach, R., Núñez, J.C., González-Pienda, J.A., y Rosário, P. (2010). Perfiles motivacionales y diferencias en variables afectivas, motivacionales y de logro. Universitas Psychologica, 9(1), 109-121.

Vélez, A.M. (1998). Aprendizaje Basado en Proyectos colaborativos en la Educación Superior. IV Congresso RIBIE, Brasilia. 